\title{
Ciudades en auge en Chile: Rol de la actividad exportadora en la dinámica del empleo urbano ${ }^{1}$
}

\begin{abstract}
Johannes Rehner. Pontificia Universidad Católica de Chile, Santiago de Chile. Sebastián Rodríguez. Pontificia Universidad Católica de Chile, Santiago de Chile. Warwick E. Murray. Victoria University, Wellington, New Zealand.
\end{abstract}

RESUMEN | Las ciudades en Chile han vivido una fase de expansión al inicio del presente siglo relacionada con actividades de exportación. Si bien un auge exportador puede producir efectos negativos sobre otros rubros, también genera importantes impulsos en las economías urbanas, debido a la demanda por servicios considerados no exportables o a través de la inversión de renta y el circuito secundario del capital. Contrastando de manera cuantitativa ciudades chilenas en auge y sin auge, se identifican diferentes explicaciones para las tendencias de empleo en tres rubros relevantes para la economía urbana. Así, en las ciudades en auge se observan importantes efectos positivos del empleo minero y de sus remuneraciones sobre el empleo en construcción y comercio. Por otro lado, en ciudades sin auge la minería pierde este rol protagónico, mientras que el gasto público y la industria no metálica, en parte procesando productos agrícolas, tienen importancia al explicar tendencias del empleo urbano.

PALABRAS CLAVE | geografía económica, economía urbana, reestructuración económica.

ABSTRACT | During the beginning of the 21st century, cities in Chile have experienced a phase of rapid expansion regarding export activities. While a booming export sector may produce negative effects on other activities, there are also important impulses on urban economies, especially through the demand for "non-tradable" services, or through investment of excessive rents and the secondary circuit of capital. By contrasting booming and non-booming cities in Chile through a quantitative exercise, we identify different explanations for employment trends in three service sectors that are highly relevant for the urban economy. In booming cities there are important positive effects due to mining employment and revenues on employment in the construction and retail sectors. On the other hand, in the non-booming cities, mining loses its outstanding role, while public spending and the non-metal industry (including agricultural products processing) are more relevant to explain urban employment.

KEYWORDS | economic geography, urban economy, economic restructuring.

Recbido el 12 de abril de 2016, aprobado el 28 de julio de 2016.

E-mails: J. Rehner, jrehner@uc.cl | S. Rodríguez, sirodri1@uc.cl | W. E. Murray, warwick.murray@vuw.ac.nz

1 Esta investigación recibió el apoyo de los proyectos Fondecyt 1150636 y Fondap 15110020 (CEDEUS). 


\section{Introducción}

Una fase prolongada de prosperidad económica provocada por el boom de las exportaciones y precios de los minerales y metales, particularmente del cobre, ha marcado el inicio del nuevo milenio en América Latina, y en particular en Chile. ${ }^{2}$ Este auge del sector exportador chileno deriva directamente de la demanda global por commodities en el siglo xxi. Se trata, sin embargo, de una bonanza que ha tenido una marcada variabilidad en el tiempo y en el espacio, favoreciendo la exportación y economía de algunas regiones mucho más que de otras (Rehner, Baeza \& Barton, 2014). Tal situación es apreciable en aquellas regiones receptoras de importantes ingresos por exportación de commodities, en las que se ha producido un vertiginoso crecimiento de las economías urbanas. Este crecimiento, a su vez, ha generado un fenómeno que se puede denominar como "ciudades en auge" ("boom towns"), concepto que será abordado en profundidad más adelante. Tales explicaciones para el surgimiento de las ciudades en auge, que remiten a la renta obtenida a partir de la exportación de minerales y metales, no descartan la posibilidad de que el auge esté dado también por otras actividades productivas exportadoras, como lo son las industrias forestal, agrícola y pesquera, las cuales, si bien no tienen el protagonismo del cobre a escala nacional, son sumamente relevantes en su contexto local y regional (Rehner \& Vergara, 2014).

El argumento central de este trabajo plantea que los territorios marcados por la extracción de commodities generan flujos de capital que se materializan en las economías urbanas, según lo planteado en la discusión del segundo circuito (Fox Gotham, 2009; Harvey, 2011). La exportación basada en recursos naturales se genera en territorios de extracción minera, agrícola, forestal y pesquera, en su mayoría alejados de las ciudades, pero su puesta en valor en los mercados globales implica la necesidad de articularse a través de nodos - funciones que cumplen las ciudades-, donde confluyen en parte los flujos materiales, pero por sobre todo las transacciones financieras, prestación de servicios y de las personas ligadas directa e indirectamente a la actividad extractiva y exportadora. Estas ciudades son, por ende, más una bisagra del flujo de commodities y capital financiero, que un centro de comando y de procesamiento. Aun así, una parte relevante de estos flujos "aterriza” en las ciudades y se materializa en efectos locales, donde el aumento rápido de los ingresos generados por la exportación es capturado en parte por las empresas y el Estado, y también por los empleados no solo en el sector exportador en auge, sino también en sectores no exportables de la economía. Se trata de un proceso explicable a partir de lo planteado en la teoría del síndrome holandés (Corden \& Neary, 1982); y en este sentido, tales efectos estarían manifestándose en características comunes de las "ciudades en auge".

El artículo se compone de una primera sección donde se definen criterios constituyentes de las ciudades en auge, basados en la reflexión teórica, y luego, en una segunda sección, se identifican áreas urbanas en Chile que muestran tales

2 En el período 2002-2015, el valor promedio anual de la exportación de cobre fue de casi 30.000 millones de dólares, aportando un 13,3\% al Producto Interno Bruto (PIB) de Chile (Comisión Chilena del Cobre [CochiLco], 2016; Banco Central de Chile, 2016). 
características. En la tercera sección se plantean explicaciones teóricas para el impacto de la exportación sobre las economías urbanas, particularmente las actividades económicas no exportadoras. A partir de esto, se presentan modelos de regresión múltiple para estimar el efecto de la exportación sobre estas actividades, en contraste con otros efectos en las ciudades en auge y en ciudades que no tienen esta característica. Finalmente, el artículo cierra con conclusiones para Chile e implicancias teóricas, profundizando en la conceptualización de los vínculos entre exportación y crecimiento urbano.

\section{“Ciudades en auge" - características y situación en Chile}

En el siguiente apartado se propone organizar diferentes ciudades del país en tres grupos, que se refieren a la condición de "auge" y no a sus consecuencias en términos sociales, ${ }^{3}$ los cuales serán discutidos posteriormente en la presente reflexión.

\section{Crecimiento como criterio definitorio de ciudades en auge}

Se puede suponer que un sector extractivo-exportador dinámico fomenta un crecimiento de la población urbana, aunque su actividad se desarrolla principalmente fuera de la ciudad. Las características particulares de las regiones con vocación minera (Tarapacá, Antofagasta, Atacama y Coquimbo), como su baja densidad poblacional y la escasa oferta de la fuerza laboral, combinadas con el efecto "crowding out" descrito por la tesis del síndrome holandés (Corden \& Neary, 1982), han generado un flujo importante de fuerza laboral hacia ellas. La escasez de fuerza laboral es suplida por la llegada de nuevos habitantes, provenientes tanto de localidades cercanas como de otras regiones del país, y por la conmutación a larga distancia, destacando en este sentido la región de Antofagasta como receptora de trabajadores (Aroca \& Atienza, 2008). En los últimos años también se ha evidenciado la llegada de inmigrantes internacionales hacia ciudades con perfil minero - por ejemplo, las de la región de Antofagasta (Taylor, 2014)-, producto del elevado nivel de sueldos que allí se ofrecen. Por sobre todo, las ciudades capitales regionales o provinciales del país, situadas en regiones-commodities (Daher, 2003), se han caracterizado por la concentración de población durante las últimas décadas, a partir del auge del modelo exportador en Chile (Huidobro \& Maragańo, 2010). Debido a esta llegada de nuevos habitantes y la resultante concentración de población, en particular las ciudades mineras han evidenciado un dinamismo de la economía urbana, registrando sobre todo un impulso significativo de la actividad constructora, lo que se traduce finalmente en un acelerado proceso de urbanización (Thodes, 2016).

Además de lo anterior, se puede suponer un aumento de la inversión en los espacios urbanos debido a la mayor liquidez financiera de los diferentes agentes

3 Los impactos ambientales de este auge no se discuten en este trabajo. Numerosos estudios han argumentado sobre los impactos de la explotación de recursos, sobre la contaminación atmosférica generada por los procesos mineros y las fundiciones, el desecamiento de salares, destrucción de glaciares y contaminación del agua, el riesgo ambiental por las condiciones de los tranques de relaves activos y abandonados, entre otros. Esto ha sido discutido tanto durante el auge (Romero \& Vásquez, 2005) como también previamente (Durán, 1990; Sánchez \& Huepe, 1995). 
económicos: de las empresas, del sector público y, finalmente, de los hogares. Por un lado, se espera que el sector privado invierta en estas ciudades debido a un aumento de la prestación de servicios a la minería, como se evidenció en la ciudad de Antofagasta (Atienza, Lufin, Soto \& Cortés, 2015, pp. 108-109). Por otro lado, el sector público, por disponer de mayores ingresos, incluso cuando se trata de un porcentaje muy reducido del valor de exportación, muestra un rápido incremento de la inversión, como ocurre, por ejemplo, en la región de Antofagasta, con un crecimiento del 12\% anual entre 2001 y 2013 (Ministerio de Desarrollo Social, 2014). Debido al mayor nivel de ingresos obtenidos por el empleo en el sector en auge, los hogares no solamente aumentan su consumo, sino que también realizan inversiones a largo plazo, como se ha evidenciado, por ejemplo, en el auge inmobiliario en la ciudad de Antofagasta, donde la inversión inmobiliaria se incrementó $600 \%$ en el período 2004-2014 (Cámara Chilena de la Construcción [cchc], 2015). En sumatoria, estas inversiones se materializan en una expansión de la mancha urbana. Esta última lectura del proceso se puede complementar con una visión diferente, basada en el argumento relativo al "circuito secundario" del capital en la economía urbana. Este circuito secundario hace referencia a la necesidad de ser invertido que presenta el capital, particularmente el vinculado al exceso de renta, lo que produce una "relación íntima entre el desarrollo del capitalismo y la urbanización” (Harvey, 2008, p. 24).

\section{La "riqueza" en las ciudades}

A continuación se plantean dos principales explicaciones de por qué en las ciudades en auge se concentra "riqueza" monetaria, observándose elevados ingresos, pero también un alto nivel de precios. Primero se constata que aquellas ciudades de rápido crecimiento de ingresos monetarios son más propensas a mostrar una cierta escasez de oferta en el mercado laboral, lo que provoca sueldos más altos y también inmigración. El nivel de ingresos con el que cuenta la mano de obra del sector en auge genera una demanda por infraestructura y servicios, lo cual hace subir los precios y el costo de la vida, dando lugar a un consumo desproporcionado asociado a la construcción de un gran número de viviendas y de infraestructura (Ennis, Finlayson \& Speering, 2013; Haslam-McKenzie \& Rowley, 2013). A esto se suma una escasez de oferta en los mercados de servicios y de inmuebles, que impulsa al alza sus precios. Es importante destacar que "riqueza" no incluye un criterio de distribución, por lo que se puede suponer una elevada probabilidad de creciente desigualdad y pobreza, dado que todos los habitantes tienen que asumir los altos precios, sin verse necesariamente beneficiados por un alto nivel de sueldo. Esto guarda relación con problemas evidenciados históricamente en comunidades mineras, donde se dan diferencias significativas de ingresos entre los nuevos trabajadores y los residentes permanentes (Williams, 1981; Barrett, 1994).

Una segunda perspectiva -muy diferente de la recién abordada argumentación relativa al funcionamiento de mercados- abarca la característica del circuito secundario del capital en la conceptualización neomarxista. Según este enfoque, las ciudades tienen un rol clave en la reproducción del capital obtenido como renta de la exportación, ya que, en una lógica propia del capitalismo y de la característica del negocio inmobiliario como un "fijo espacial" del excedente producido por otra 
actividad, ellas absorben lo que en la lectura neomarxista es capital "sobreacumulado" (Harvey, 2011, p. 5). En esta lectura, las ciudades son fuentes de la acumulación de capital, pero también una barrera para la movilidad del mismo (Fox Gotham, 2009, p. 356), debido al hecho de que así el capital está fijado en el espacio. Esto es un elemento clave para entender el capital como un proceso y no una cosa (Harvey, 2011 , p. 6), siendo entonces el flujo del excedente hacia las urbes, para ser allí reproducido a través de los mercados inmobiliarios, uno de los flujos constituyentes del capitalismo contemporáneo. Como consecuencia, se puede suponer que en este circuito secundario del capital los precios se impulsan hacia arriba, ya que con una cierta abundancia de capital a escala local, se aumenta la capacidad de pago no solamente de las empresas que obtienen dicha renta, sino también de los empleados.

\section{El recambio en las ciudades en auge}

Durante el siglo xIx y gran parte del xx, la lógica de funcionamiento de la extracción minera del país estuvo caracterizada por el modelo de "enclaves productivos", configurándose como una de sus dimensiones de mayor relevancia los asentamientos llamados company-towns (Alvear, 1975; Barrera, 1973; Zapata, 1977). Construidos ex novo con el propósito de conseguir la máxima concentración de capital y trabajo mediante la articulación de viviendas, equipamientos y edificios industriales de manera planificada, dichos asentamientos representaron un modelo urbano alternativo a la ciudad tradicional, con Chuquicamata (región de Antofagasta) y El Salvador (región de Atacama) como ejemplos prominentes. Los company-towns se configuraron como un espacio de trabajo que representó un polo de atracción de mano de obra, pero a la vez eran territorios controlados y desarrollados por empresas (públicas o privadas) con una organización que incorporó elementos de la vida y estructura familiar de los trabajadores (Garcés, 2003).

Desde finales del siglo xx, sin embargo, este modelo se ha visto desarticulado, como efecto de la transformación posfordista de la economía de servicios asociada a la producción y a la reproducción de la fuerza de trabajo, la tecnificación-automatización y robotización de las labores, la fragmentación productiva, la flexibilización de los regímenes de trabajo y los modelos de subcontratación (Agacino, González \& Rojas, 1998; Echeverría \& López, 2004; Sennett, 2009). En este contexto, el funcionamiento de la industria minera en el país ha pasado de estar caracterizado por los company-towns, a lo que actualmente son las ciudades mineras (Zapata, 2001; Lagos, Blanco, Torres \& Bustos, 2001).

El período de auge exportador y la nueva lógica de organización laboral han provocado cambios en la composición de la población de las ciudades mineras, atrayendo población inmigrante que se emplea en sectores como la construcción y el comercio (El Mercurio de Antofagasta, 2011), y también trabajadores provenientes de otras regiones del país, que están poco tiempo en la ciudad en que trabajan para luego volver a la ciudad de donde provienen. Esta dinámica guarda relación con la conmutación a larga distancia, la cual es entendida como el viaje de ida y vuelta que el trabajador realiza entre su residencia y su lugar de trabajo (Aroca $\&$ Atienza, 2008). Tal fenómeno ha sido estudiado en países como Canadá (Storey \& Shrimpton, 1988), Australia (Houghton, 1993; Storey, 2001, 2009), Rusia 
(Spies, 2006) y en Chile (Aroca \& Atienza, 2008; Jamett \& Paredes, 2013). En este último se ha evidenciado la existencia de un particular patrón espacial de la conmutación, ya que las regiones de los extremos norte y sur del país, y la Región Metropolitana, poseen saldos netos de conmutación positivos, mientras que las regiones del centro presentan saldos netos negativos (Aroca \& Atienza, 2008).

Sumado a lo anterior, un elemento propio de la lógica de la conmutación interregional corresponde a los viajes por trabajo realizados en avión, lo que se denomina como fly in-fly out (MacIntosh, 2012). Este término fue usado para referirse al traslado de los trabajadores en avión en las plataformas petrolíferas del golfo de México y los estudios de las actividades extractivas en Canadá y Australia, a modo de alternativa a la construcción de campamentos próximos a los yacimientos en explotación (Bell \& Brown, 2006; Storey, 2001). Entre las características del fly in-fly out se encuentran los sistemas de trabajo concentrados por turnos, en los que el trabajador permanece en el lugar de trabajo durante un número de días consecutivos (Jamett \& Paredes, 20I3), una práctica con importantes impactos sociales: se ha argumentado sobre problemas sociales derivados del recambio en las ciudades como producto de la movilidad de la fuerza laboral, y su impacto a nivel local en pequeñas localidades o boom towns, cuando estas viven una fase de auge o crisis económica (Park \& Stokowski, 2009; Wilkinson, Reynolds, Thompson \& Ostresh, 1982). Se plantea que en estas comunidades se desarrollan perturbaciones sociales que podrían llevar a una ruptura de las estructuras sociales existentes, materializada en una clara diferenciación entre los recién llegados y los habitantes permanentes de la comunidad. Estos grupos no solo ocuparían redes sociales diferentes, sino que a los nuevos residentes se les asociarían elementos negativos, como la despreocupación por el pueblo y la codicia (Howitt, 2001; Lawrie, Tonts \& Plummer, 2011; Lozeva \& Marinova, 2010).

En consecuencia, se propone resumir los siguientes criterios para identificar "ciudades en auge":

a. Crecimiento: las ciudades en auge se caracterizan (AI) por su vertiginoso crecimiento poblacional, por lo que se plantea que también presentan (A2) crecimiento económico (nivel de producción y cantidad de puestos laborales, debido a lo atractivo del mercado laboral); y, por último, (A3) aumento de la superficie del área urbana en términos de cantidad de viviendas, infraestructura, áreas comerciales, entre otros.

b. Riqueza: las ciudades en auge se caracterizan por un (B I) alto nivel de sueldo no solamente en el rubro exportador, sino también en la economía local en general; у (в2) un elevado crecimiento de la remuneración debido a escasez en la oferta de fuerza laboral. (в3) En consecuencia, se puede suponer un elevado nivel de precios en las ciudades en auge.

c. Recambio: las ciudades en auge se caracterizan por una elevada velocidad de cambio en cuanto a su composición. (CI) Un porcentaje relevante de su población está constituido por inmigrantes, debido a la escasez de oferta laboral a escala local y el nivel de sueldo elevado. (c2) Hay una elevada conmutación a larga distancia, 
frecuentemente por vía aérea ("fly in-fly out"), lo que significa que una parte relevante de la fuerza laboral a escala local va cambiando a muy corto plazo.

\section{Identificando "ciudades en auge" en Chile}

Cada uno de los tres criterios que caracterizan a las ciudades en auge se representa en un conjunto de variables (tabla 1), para el período que comprende los años 2005 a 2013. La elección de este período permite conocer tres fases: la primera, de auge exportador (2005-2008); una segunda fase marcada por la crisis subprime 2008-2009; y por último, una tercera fase de recuperación desde el año 2010 en adelante. El criterio relacionado con el crecimiento de las ciudades está representado por las siguientes variables: incremento poblacional (AI), aumento del empleo (A2) y expansión de la superficie urbana (A3). La riqueza en las ciudades en auge está representada por el nivel de sueldos (вI) en dos momentos diferentes, nivel que se calculó con base en las remuneraciones de los trabajadores dependientes del sector comercio, el aumento de estas remuneraciones (в2) y el nivel de precios inmobiliarios (в3), el cual se determinó a su vez a partir del costo del arriendo de una vivienda. El criterio referido al recambio en las ciudades está representado por la tasa de inmigrantes en las ciudades respectivas (CI) y el crecimiento del número de pasajeros llegados en avión (c2). De esta forma, se muestran los datos de diez ciudades en Chile, cinco de la zona norte y cinco del sur del país, las que permiten identificar cuáles de estas ciudades pueden ser consideradas en auge.

Los datos muestran que cuatro ciudades del norte-Antofagasta, Calama, Copiapó y la conurbación La Serena-Coquimbo- presentan tendencias que permiten considerarlas ciudades en auge. En general, durante el período 2005-2013 estas ciudades experimentaron un crecimiento significativo en su población y llegada de pasajeros en avión, y tienen una elevada tasa de migrantes, evidenciando su característica de polos de atracción de personas. Asimismo, la generación de nuevos empleos y las remuneraciones también tuvieron un crecimiento importante, lo que contrasta con el alto nivel de precios en estas ciudades en comparación con el promedio nacional.

Caso contrario, se identifican ciudades que no se encuentran en una situación generalizada de auge, tales como Iquique en el norte y ciudades del sur como Talca, Chillán, Los Ángeles, Concepción y Temuco. Estas, si bien en determinadas variables muestran una situación de auge, para el resto de los indicadores registran en general valores que no superan el promedio nacional; por ende, con ellas se plantea un panorama de ciudades sin auge. Sin embargo, cabe mencionar el caso de Temuco, ya que presentó el mayor crecimiento del empleo en este período, lo cual contrasta con que es la ciudad que tiene el crecimiento más bajo en remuneraciones, con un aumento de población levemente superior al promedio nacional y escasa llegada de inmigrantes. 


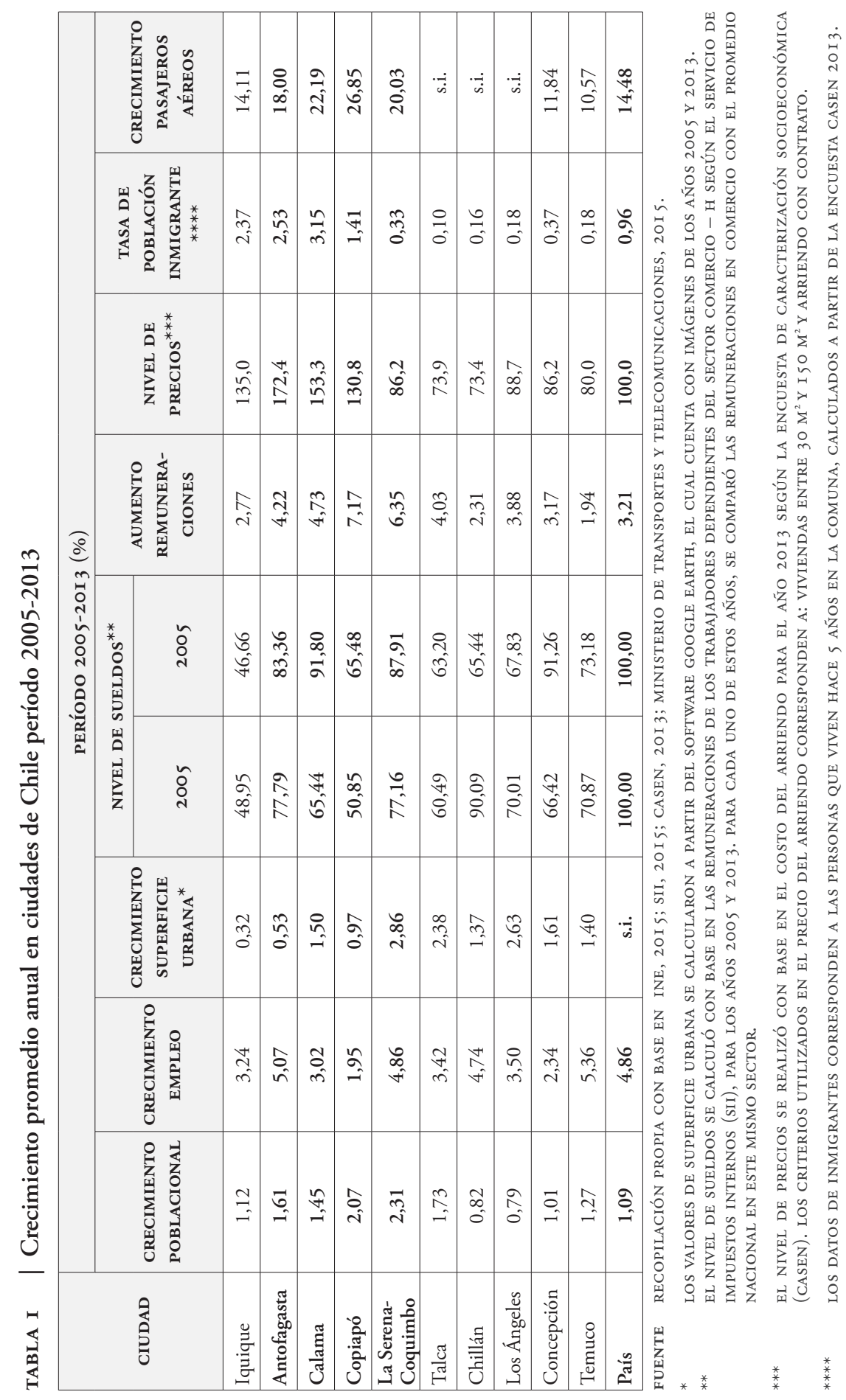




\section{El impacto del auge exportador en las economías urbanas en los rubros no exportadores}

La literatura especializada ha argumentado sobre cómo los ingresos económicos generados en Chile por exportación de commodities y el crecimiento económico a nivel nacional han reestructurado los mercados laborales urbanos, impactando en el empleo y las remuneraciones de manera diferenciada por rubro y ciudad (De Mattos, 2002; Fuentes \& Link, 2014; Rehner \& Vergara, 2014). Para explicar estas tendencias, resulta fundamental referirse a la denominada tesis del "síndrome holandés" (Corden \& Neary, 1982), la cual discute el efecto que tiene un auge exportador en la economía de un país. Se argumenta que, cuando un sector exportador en particular entra en una fase de auge debido al aumento del precio de exportación, al descubrimiento de nuevos yacimientos o por el uso de nuevas tecnologías, ello tiene efectos negativos sobre la exportación de otros bienes, provocando un menor crecimiento en estos sectores económicos rezagados (Van Wijnbergen, 1984; Corden, 1984). Sin embargo, lo más relevante para la presente discusión no es este efecto que frena otros sectores exportadores, sino la pregunta respecto de cómo este crecimiento dinámico de la exportación impacta en los sectores no exportadores, los "non-tradables" (Jeftanovic, 2010).

Se puede suponer que los ingresos generados por el sector exportador fomentan el consumo de los hogares. Esto es particularmente relevante para las ciudades, ya que en ellas se concentran rubros que pueden ser considerados "no exportables", como los servicios al consumidor final, el comercio y la gastronomía, entre otros. También se puede considerar que los hogares beneficiados por el ingreso a raíz de un auge exportador, además de su consumo, aumentan la inversión, por ejemplo en la adquisición de una vivienda o la remodelación de existentes. Esto depende evidentemente de la parte de los ingresos obtenidos por los trabajadores del sector en auge que se gastan en las ciudades próximas a las actividades extractivas. Debido a la conmutación a larga distancia en la minería, se puede suponer que una gran parte de tales ingresos se desembolsa en otra región, alejada de la actividad minera (Aroca \& Atienza 2008).

Siguiendo en esta lógica, el sector empresarial también genera efectos sobre los "non tradables", principalmente por la inversión, tomando en cuenta -por ejemploque la mayor parte de la inversión en minería se gasta en actividades de construcción. Además, el mismo sector en auge genera consumo, por ejemplo en viajes de negocio, el alojamiento y consumo de los prestadores de servicios especializados, subcontratistas, entre otros.

En consecuencia, para analizar la pregunta sobre el efecto del auge exportador en la economía urbana, nos enfocamos en el ámbito de los non-tradables, destacando tres rubros directamente relacionados con el gasto de consumo y de inversión de los diferentes agentes: el comercio, la construcción y la hotelería/restaurantes. En el centro de interés de este artículo no está la pregunta sobre si estos sectores basados en el auge exportador pudieron mejorar sus ventas o ganancias; lo que interesa es, más bien, la importancia de estas actividades de servicio para el empleo en la ciudad y el ingreso para los hogares urbanos. 
Como punto de partida, resulta relevante reconocer que la fase de auge exportador se asocia a un aumento del empleo y las remuneraciones en los rubros de construcción, comercio y hotelería/restaurantes. En las ciudades analizadas, los tres rubros presentaron un importante saldo positivo en la tendencia del empleo en este período, a excepción del rubro hotelería/restaurantes en la ciudad de Los Ángeles (tabla 2).

TABLA 2 Crecimiento promedio anual del empleo según rubro no exportador

\begin{tabular}{|l|c|c|c|c|c|c|}
\hline \multirow{2}{*}{ CIUDAD } & \multicolumn{2}{|c|}{ PERÍODO 2005-20I 3 (\%) } & \multicolumn{3}{c|}{ AÑO CRISIS 2009 (\%) } \\
\cline { 2 - 7 } & CONSTRUCCIÓN & COMERCIO & $\begin{array}{c}\text { HOTELERÍA / } \\
\text { RESTAURANTES }\end{array}$ & CONSTRUCCIÓN & COMERCIO & $\begin{array}{c}\text { HOTELERÍA / } \\
\text { RESTAURANTES }\end{array}$ \\
\hline Iquique & 0,63 & 3,99 & 3,46 & 0,91 & $-3,43$ & $-3,14$ \\
\hline Antofagasta & 5,38 & 2,74 & 7,01 & $-3,87$ & $-18,15$ & 21,78 \\
\hline Calama & 3,06 & 0,75 & 3,40 & $-10,91$ & 1,95 & $-13,44$ \\
\hline Copiapó & 4,68 & 3,55 & 4,40 & $-1,09$ & $-7,41$ & $-1,37$ \\
\hline $\begin{array}{l}\text { La Serena - } \\
\text { Coquimbo }\end{array}$ & 9,79 & 4,01 & 11,93 & $-21,98$ & $-8,75$ & 11,13 \\
\hline Talca & 1,67 & 3,79 & 8,76 & $-5,68$ & $-7,87$ & $-1,75$ \\
\hline Chillán & 5,54 & 3,68 & 1,24 & $-13,55$ & $-11,32$ & $-21,63$ \\
\hline Los Ángeles & 5,39 & 3,98 & $-10,30$ & $-4,17$ & $-1,40$ & 22,20 \\
\hline Concepción & 1,47 & 4,52 & 10,37 & $-14,99$ & 4,81 & 20,31 \\
\hline Temuco & 3,73 & 3,63 & 3,02 & $-9,62$ & $-6,19$ & $-13,94$ \\
\hline $\begin{array}{l}\text { Total 10 } \\
\text { ciudades }\end{array}$ & 3,60 & 3,79 & 4,53 & $-10,23$ & $-4,56$ & 4,64 \\
\hline
\end{tabular}

FUENTE ELABORACIÓN PROPIA CON BASE EN DATOS OBTENIDOS DEL SII, 20 I 5

Respecto a las remuneraciones el panorama es similar, ya que en gran parte de las ciudades los tres rubros experimentaron un crecimiento positivo, a excepción de comercio y hotelería/restaurantes en la ciudad de Chillán, y también comercio en Los Ángeles, los que presentaron una tendencia negativa (tabla 3).

Asimismo, la crisis subprime del ańo 2009 permite conocer de qué manera responden los tres rubros a un año marcado por la crisis. Al respecto, se observa que, en tal situación, construcción y comercio experimentaron una reducción del empleo, mientras que hotelería/restaurantes pareció no verse afectado en general por la crisis, mostrando tendencias disparejas en las diez ciudades. Respecto a los impactos de la crisis sobre las remuneraciones, ella parece no causar un efecto negativo, sino que, al contrario, las remuneraciones siguieron experimentando un crecimiento positivo en los tres rubros no exportadores, siendo el de hotelería/restaurantes el que presentó la mayor variación para el año 2009. 
TABLA 3 | Crecimiento promedio anual de remuneraciones según rubro no exportador

\begin{tabular}{|l|c|c|c|c|c|c|}
\hline \multirow{2}{*}{ CIUDAD } & \multicolumn{2}{|c|}{ PERÍODO 2005-20I3 (\%) } & \multicolumn{3}{c|}{ AÑO CRISIS 2009 (\%) } \\
\cline { 2 - 7 } & CONSTRUCCIÓN & COMERCIO & $\begin{array}{c}\text { HOTELERÍA / } \\
\text { RESTAURANTES }\end{array}$ & CONSTRUCCIÓN & COMERCIO & $\begin{array}{c}\text { HOTELERÍA / } \\
\text { RESTAURANTES }\end{array}$ \\
\hline Iquique & 5,20 & 2,37 & 3,21 & 3,11 & 7,40 & 7,97 \\
\hline Antofagasta & 6,51 & 3,88 & 4,78 & 8,73 & 19,64 & 6,81 \\
\hline Calama & 5,57 & 7,43 & 5,64 & 19,96 & 16,99 & 18,32 \\
\hline Copiapó & 12,02 & 6,29 & 4,20 & 15,00 & 8,80 & 21,44 \\
\hline $\begin{array}{l}\text { La Serena - } \\
\text { Coquimbo }\end{array}$ & 8,87 & 4,68 & 4,31 & 2,97 & 12,80 & 11,29 \\
\hline Talca & 6,14 & 3,55 & 0,59 & 4,26 & 12,13 & 14,10 \\
\hline Chillán & 5,70 & $-1,05$ & $-0,67$ & 4,54 & 12,84 & 7,19 \\
\hline Los Ángeles & 5,98 & $-0,18$ & 5,57 & 8,26 & 15,32 & 8,91 \\
\hline Concepción & 4,21 & 7,16 & 4,30 & 7,33 & 3,19 & 27,24 \\
\hline Temuco & 3,72 & 3,40 & 1,66 & 1,78 & 13,21 & 12,54 \\
\hline $\begin{array}{l}\text { Total 10 } \\
\text { ciudades }\end{array}$ & $\mathbf{6 , 6 0}$ & 4,05 & 3,29 & 9,31 & 12,45 & 13,69 \\
\hline
\end{tabular}

FUENTE ELABORACIÓN PROPIA CON BASE EN DATOS OBTENIDOS DEL SII, 20 I 5

\section{Metodología}

La metodología empleada a continuación busca identificar en qué medida el crecimiento del empleo en sectores "no exportables", depende de otras variables. Para ello, con el software spss 20 se construyeron modelos de regresión (Ordinary Least Square, ols) para el crecimiento del empleo en cada uno de los rubros no exportables elegidos (construcción, comercio y hotelería/restaurantes). El modelo aplicado es logarítmico, considerando el logaritmo natural de cada una de las variables para evitar problemas asociados a la distribución de los valores (heterocedasticidad). La temporalidad de los datos comprende los años 2005-2013, período que permite incluir en el análisis el auge exportador (2005-2008), la crisis subprime del año 2009 y, por último, la fase de recuperación desde el año 2010 en adelante.

Como fuente principal se utilizó la base de datos del Servicio de Impuestos Internos (sII), la cual informa el número de trabajadores dependientes y remuneraciones a escala comunal y diferenciada por actividad económica. Se debe enfatizar que el empleo, según los datos del siı, se registra en el lugar donde está contribuyendo la empresa (casa matriz) y se refiere solamente a los empleados dependientes. Sumado a esto, se trabajó con la base de datos de Aduanas Chile, que incluye valores de exportación regional diferenciados por productos. La desagregación por rubros sigue la clasificación ciıU (Clasificación Internacional Industrial Uniforme) agregada a nivel de sector económico (dos dígitos). Otras variables incluidas en este trabajo corresponden a la información sobre la inversión nacional y la extranjera a escala regional, obtenida desde las páginas web del Banco Central y del Comité de 
Inversión Extranjera, respectivamente. Asimismo, se ingresó información sobre la inversión gubernamental a escala de ciudad, obtenida de distintos ministerios y de las municipalidades respectivas. Los datos correspondientes a las variables asociados al turismo, como el número de llegada de turistas, número de noches que pasan los turistas y los ingresos por pernoctación a escala regional, fueron obtenidos del Instituto Nacional de Estadísticas (INE). Por último, se consideran datos sobre comercio local, como el número de actividades económicas y venta de las empresas diferenciadas por actividad económica, ambas obtenidas del sir. Sumado a esto, se utilizó información referente a las ventas de los supermercados a escala regional, obtenidas del Observatorio Regional de la Subsecretaría de Desarrollo Regional y Administrativo (subDERE).

Los datos fueron procesados a escala urbana, correspondientes a Iquique, Antofagasta, Calama, Copiapó, la conurbación la Serena-Coquimbo, Talca, Chillán, Los Ángeles, Concepción y Temuco (figura 1). Cabe destacar que, en algunos casos, estas ciudades se conforman por una sola comuna (por ejemplo, Antofagasta) y en otros, por una agrupación de comunas relacionadas funcionalmente (por ejemplo Concepción), de acuerdo con la clasificación de áreas urbanas propuesta por la Organización para la Cooperación y el Desarrollo Económicos (OCDE, 2012). ${ }^{4}$ Cada una de las variables consideradas en los modelos es expresada como tasa de crecimiento. De esta forma, los modelos estadísticos se calculan con los datos de una determinada variable para cada una de las ciudades analizadas en cada uno de los años en cuestión (2005-2013). Los modelos para ciudades en auge consideran 32 casos (cuatro ciudades, ocho años cada una) y los de ciudades sin auge están construidos con 40 casos (cinco ciudades, ocho años). Los resultados consideran solo modelos de regresión con un valor explicativo satisfactorio $\left(\mathrm{R}^{2}>0,5\right)$, con buena significancia (al nivel de 0,005 ) y sin problemas de colinealidad (tolerancia y VIF satisfactorio).

En un primer análisis, los resultados de los modelos de regresión realizados para las ciudades sin auge no mostraron valores satisfactorios, ya que no cumplieron con la condición $\mathrm{R}^{2}>0,5$ y mostraron niveles de significancia que no podrían ser considerados estadísticamente válidos. Entre las causas que posiblemente explicarían esto se encuentra la falta de homogeneidad del grupo de seis ciudades sin auge, particularmente el hecho de que cinco de ellas se localizan geográficamente en la zona sur del país y solo Iquique se encuentra en el norte minero del país. De esta forma, no fue posible encontrar patrones en común en los modelos referidos a estas seis ciudades; el empleo en los tres sectores no exportadores parece responder de forma diferente en las seis ciudades sin auge incluidas en el análisis, debido posiblemente a la dispar localización y base económica de estas ciudades. Para evitar este problema, los modelos de regresión para las ciudades sin auge consideraron Concepción, Temuco, Chillán, Los Ángeles y Talca, exceptuando de este grupo a la ciudad de Iquique.

Esta metodología permite identificar qué comunas se deben considerar parte de una ciudad en términos funcionales, aplicando criterios de movilidad laboral, densidad poblacional, población total y población que vive dentro de la ciudad (oCDE, 2012). 
FIGURA I | Ciudades analizadas y valor de la exportación regional para el año 2013

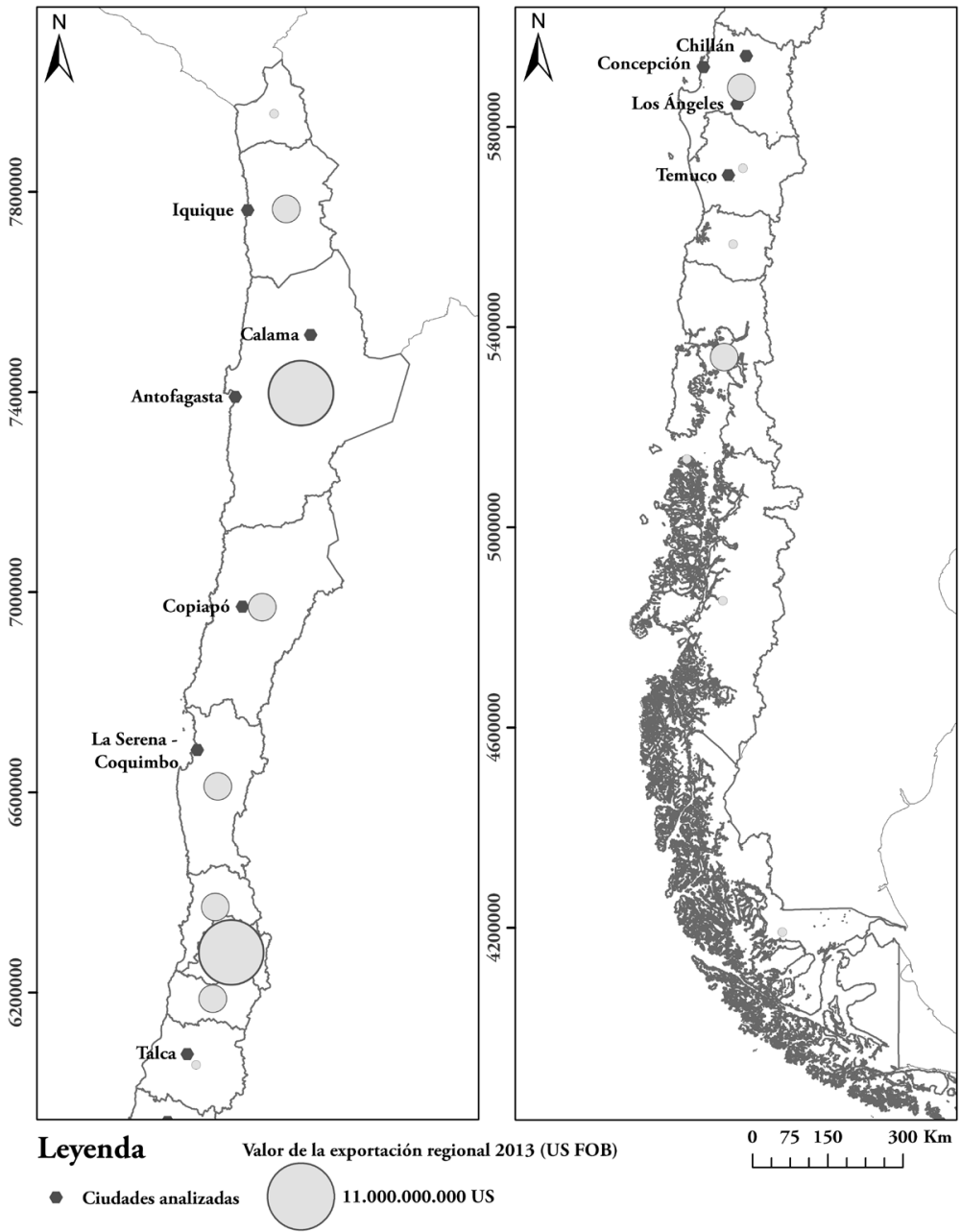

FUENTE ELABORACIÓN PROPIA

Resultados: Explicaciones de las tendencias del empleo urbano en los rubros no exportadores

A continuación se discuten los resultados de los modelos de regresión (oLs) para el empleo en cado uno de los tres rubros analizados, contrastando los modelos para ciudades en auge con aquellos de las ciudades sin auge (3+3 modelos, véase tabla 4). El análisis se encuentra estructurado con base en grupos de variables, correspondientes a los temas exportación, inversión, empleo, remuneraciones, consumo, emprendimiento y turismo. 


\section{La actividad exportadora}

Se espera que el auge exportador tenga efecto sobre el crecimiento del empleo en rubros no exportadores, por la demanda de las empresas extractivas y debido a la demanda generada por el consumo de los trabajadores. De acuerdo con esto, los resultados muestran que, en las ciudades en auge, la actividad exportadora asociada a la minería actúa en cierto grado como motor de crecimiento del empleo en los rubros no exportadores de construcción y comercio $\left(\beta_{\text {est. }}=0,418\right.$ y 0,344 respectivamente). Sin embargo, esta influencia tiene una baja significancia (a nivel 0,1 ). Por el contrario, el empleo en el rubro de hotelería/restaurantes se asocia de forma negativa con la exportación minera $\left(\beta_{\text {est. }}=-0,310\right)$, con una lógica de funcionamiento propia, diferente a las tendencias presentes en los otros dos rubros. En cuanto al panorama de las ciudades sin auge, los resultados muestran que la exportación agrícola no tiene relación con el empleo en sectores "non tradable". La exportación de la industria no metálica actúa impulsando el crecimiento del empleo en comercio $\left(\beta_{\text {est. }}=0,825\right)$, pero no en los demás rubros, mientras que la exportación asociada a la minería tiene un impacto inverso sobre el comercio $\left(\beta_{\text {est. }}=-0,808\right)$.

\section{La inversión nacional y extranjera}

A partir de las reflexiones teóricas, se espera que la inversión tenga efectos secundarios positivos sobre el empleo en servicios, sobre todo en construcción, debido a que se supone que una mayor inversión se materializa en la construcción de infraestructura, vivienda y edificios comerciales. Además, se pueden suponer efectos secundarios sobre el consumo y la hotelería, bajo el supuesto de que una mayor inversión traería más personas que demandan estos servicios. Y por último, un aumento de la inversión podría tener efectos indirectos, mejorando las condiciones para el desarrollo de todas las actividades de servicios, por ejemplo debido a una mejor infraestructura. Los resultados son contradictorios. En las ciudades en auge la inversión demuestra poca influencia, y en aquellos modelos en los cuales se evidencia un cierto nivel de influencia (la inversión total influye en empleo en construcción y la inversión a través del Fondo Nacional de Desarrollo Regional [FNDR], en el empleo en comercio), esta es negativa, sugiriendo un comportamiento contracíclico de la inversión pública. Esta última observación se explica posiblemente por el hecho de que en las ciudades con mayor crecimiento económico, el aumento de la inversión pública es relativamente lento. En general, se puede constatar que el papel de la inversión es relativamente reducido en comparación con el efecto del valor de la exportación.

Por su parte, el panorama de las ciudades sin auge muestra una imagen muy distinta, dejando en evidencia que el conjunto de variables asociadas a la inversión pública doméstica en diferentes escalas se relaciona de manera diferenciada con los tres rubros analizados. La inversión pública total muestra influencia positiva, pero débil, sobre el empleo en gastronomía; e influencia negativa sobre el empleo en comercio $\left(\beta_{\text {est. }}=-0,789\right)$; la inversión a través del FNDR actúa impulsando de forma importante el crecimiento del empleo en comercio $\left(\beta_{\text {est. }}=0,642\right)$; y por último, la inversión pública municipal se relaciona de forma positiva con el crecimiento del empleo en hotelería/restaurantes $\left(\beta_{\text {est. }}=0,435\right)$. 
TABLA 4 Modelos de regresión - empleo en las ciudades en auge y sin auge

\begin{tabular}{|c|c|c|c|c|c|c|}
\hline \multirow[b]{2}{*}{ VARIABLES } & \multicolumn{3}{|c|}{ CIUDADES EN AUGE } & \multicolumn{3}{|c|}{ CIUDADES SIN AUGE } \\
\hline & $\begin{array}{c}\text { EMPLEO } \\
\text { CONSTRUC- } \\
\text { CIÓN } \\
\end{array}$ & COMERCIO & $\begin{array}{c}\text { EMPLEO } \\
\text { HOTELERÍA / } \\
\text { RESTAURANTES }\end{array}$ & \begin{tabular}{|c|} 
EMPLEO \\
CONSTRUC- \\
CIÓN \\
\end{tabular} & $\begin{array}{l}\text { EMPLEO } \\
\text { COMERCIO }\end{array}$ & $\begin{array}{c}\text { EMPLEO } \\
\text { HOTELERÍA / } \\
\text { RESTAURANTES } \\
\end{array}$ \\
\hline $\begin{array}{l}\text { Valor de la exportación en } \\
\text { agricultura }\end{array}$ & & & & $(0,504)$ & & $(0,291)$ \\
\hline $\begin{array}{l}\text { Valor de la exportación en } \\
\text { minería }\end{array}$ & 0,418 & 0,344 & $-0,310$ & $(-0,254)$ & $-0,808^{* * *}$ & $(0,195)$ \\
\hline $\begin{array}{l}\text { Valor de la exportación en } \\
\text { industria no metálica }\end{array}$ & & & & $(-0,321)$ & $0,825^{* * *}$ & \\
\hline Inversión pública total & $-0,539^{*}$ & $(-0,297)$ & 0,284 & $(0,268)$ & $-0,789^{* * *}$ & $0,380^{*}$ \\
\hline Inversión pública regional & & $(0,419)$ & & $(0,360)$ & & \\
\hline Inversión pública FNDR & & $-0,443^{*}$ & & & $0,642^{* *}$ & \\
\hline Inversión pública municipal & & & & & & $0,435^{*}$ \\
\hline PIB agricultura & & & & $0,492^{*}$ & & \\
\hline PIB minería & $-0,339$ & & & & & \\
\hline Empleo en minería & $0,438^{*}$ & $-0,250$ & & & & \\
\hline $\begin{array}{l}\text { Empleo en industria no } \\
\text { metálica }\end{array}$ & & $0,307^{*}$ & & 0,266 & $0,501^{*}$ & $-0,811^{* * *}$ \\
\hline Empleo en construcción & & & $0,616^{* * *}$ & & & \\
\hline $\begin{array}{l}\text { Empleo en hotelería/restau- } \\
\text { rantes }\end{array}$ & $0,761^{* * *}$ & & & & 0,373 & \\
\hline Remuneraciones en minería & $0,441^{*}$ & & & & & \\
\hline $\begin{array}{l}\text { Remuneraciones en industria } \\
\text { metálica }\end{array}$ & & & $(-0,211)$ & & & \\
\hline $\begin{array}{l}\text { Remuneraciones en } \\
\text { construcción }\end{array}$ & $-0,238$ & & $(-0,231)$ & & & \\
\hline $\begin{array}{l}\text { Remuneraciones en hotelería/ } \\
\text { restaurantes }\end{array}$ & & & & & $0,514^{* *}$ & \\
\hline Ingresos por noche de turistas & & & $0,368^{* *}$ & & & \\
\hline $\begin{array}{l}\text { Valor de ventas de } \\
\text { supermercados }\end{array}$ & & $0,563^{* *}$ & & & & \\
\hline Valor de ventas, construcción & & & & $0,442^{*}$ & & \\
\hline $\begin{array}{l}\mathrm{N}^{\circ} \text { Contribuyentes Inicio de } \\
\text { Actividades minería }\end{array}$ & $-0,194$ & & & & & \\
\hline $\begin{array}{l}\mathrm{N}^{\circ} \text { de actividades económicas } \\
\text { minería }\end{array}$ & $(-0,166)$ & & & & & \\
\hline $\begin{array}{l}\mathrm{N}^{\circ} \text { de actividades económicas } \\
\text { construcción }\end{array}$ & $(-0,215)$ & & & & & \\
\hline $\begin{array}{l}\mathrm{N}^{\circ} \text { de actividades económicas } \\
\text { comercio }\end{array}$ & & $0,440^{* *}$ & & & & \\
\hline $\mathrm{R}^{2}$ corregido & 0,510 & 0,539 & 0,574 & 0,556 & 0,595 & 0,460 \\
\hline
\end{tabular}

FUENTE ELABORACIÓN PROPIA

NOTA LOS VALORES QUE SE MUESTRAN CORRESPONDEN AL BETA ESTANDARIZADO ( $\beta$ EST.). ASIMISMO, SE DAN A CONOCER SOLAMENTE MODELOS CON VALORES SIGNIFICATIVOS (AL NIVEL O,O5O). EL R2 CORREGIDO DE CADA UNO DE LOS MODELOS DEMUESTRA EL GRADO DE DETERMINACIÓN DEL MODELO DE REGRESIÓN CONSTRUIDO. EL TEST DE COLINEALIDAD MUESTRA VALORES SATISFACTORIOS: TOLERANCIA >O, I; FIV <7, O EN TODOS LOS CASOS. RESPECTO A LA SIGNIFICANCIA DE CADA VARIABLE, EN NEGRITA SE ENCUENTRAN VALORES SIGNIFICATIVOS (NIVEL O,I) Y EN ESPECÍ́FICO, SE DESTACA SI EL VALOR ES < A O,0 5 CON UNA *, CON ** SI ES < A O,OI Y CON *** SI EL NIVEL DE SIGNIFICANCIA ES < A O,OOI. ADEMÁS, EXISTEN VALORES ENTRE PARÉNTESIS QUE SE CARACTERIZAN POR TENER UNA SIGNIFICANCIA QUE NO CUMPLE CON LOS CRITERIOS DESEADOS, LOS CUALES NO FUERON INTERPRETADOS EN EL ANÁLISIS POSTERIOR, SIN EMBARGO SE DECIDIÓ NO ELIMINAR LAS DEL MODELO YA QUE CONTRIBUYEN A LA EXPLICACIÓN GENERAL DE CADA UNO DE LOS SEIS MODELOS PRESENTADOS. 


\section{El empleo y las remuneraciones}

Como producto de los elevados ingresos que reciben los trabajadores del sector en auge, se espera que el consumo y gasto por parte de ellos tenga efectos en otros sectores, como los servicios, en términos de empleo y remuneraciones. Con base en esto, los resultados muestran que, en las ciudades en auge, el empleo en minería actúa impulsando el crecimiento del empleo en construcción $\left(\beta_{\text {est. }}=0,438\right)$, tal como lo hacen las remuneraciones $\left(\beta_{\text {est. }}=0,441\right)$. Adicionalmente, es posible observar que el mayor impacto positivo sobre el empleo en construcción lo ejerce el empleo en hotelería/restaurantes $\left(\beta_{\text {est }}=0,761\right)$ y viceversa, el empleo en hotelería/restaurantes depende fuertemente del empleo en construcción $\left(\beta_{\text {est. }}=0,616\right)$.

Respecto a las ciudades sin auge, los resultados muestran que el empleo asociado a la industria no metálica cumple un rol importante, ya que, por un lado, impulsa el crecimiento del empleo en construcción y comercio $\left(\beta_{\text {est. }}=0,266\right.$ y $\left.\beta_{\text {est. }}=0,501\right)$, mientras que, por otro lado, se relaciona de manera negativa con el crecimiento del empleo en hotelería/restaurantes $\left(\beta_{\text {est. }}=-0,811\right)$. Asimismo, se evidencia que las remuneraciones en hotelería/restaurantes impactan positivamente sobre el empleo en comercio $\left(\beta_{\text {est. }}=0,514\right)$.

\section{Consumo, emprendimiento y turismo}

A modo de control se ingresaron otras variables en el modelo, como son las ventas en los diferentes rubros, la cantidad de empresas por rubro y algunos indicadores asociados al turismo. Estas variables ofrecen un aporte puntual a los modelos, revelando que, de acuerdo con lo esperable, el aumento en la venta por parte de los supermercados actúa impulsando el crecimiento del empleo en comercio $\left(\beta_{\text {est. }}=0,563\right)$ y los ingresos por pernoctaciones generan empleo en gastronomía $\left(\beta_{\text {est. }}=0,368\right)$, según lo observado en las ciudades en auge. Asimismo, el empleo en construcción se explica en las ciudades sin auge por un aumento de las ventas en este mismo rubro $\left(\beta_{\text {est. }}=0,442\right)$.

Por otra parte, se esperaría que el emprendimiento asociado posiblemente a la liquidez derivada del auge exportador y la llegada de visitantes, ya sea por ocio o viajes de trabajo, impulsaría la demanda por servicios no exportables. Los resultados, sin embargo, muestran que el emprendimiento en general tiene poca relación con las variables de empleo, con la salvedad del aumento de actividades en comercio, el cual tiene efectos positivos sobre el empleo en este mismo sector en las ciudades en auge $\left(\beta_{\text {est. }}=0,440\right)$.

\section{Ciudades en auge vs. ciudades sin auge}

Si bien en ambos tipos de ciudades la actividad exportadora y la inversión desempeñan un rol clave en la dinámica del empleo en los rubros no exportadores al actuar como motor o freno de la economía urbana, según ciudad y rubro, existen tendencias que permiten sostener el argumento sobre las diferencias entre las ciudades en auge y las demás ciudades. En términos generales, los resultados muestran que la actividad exportadora y la inversión nacional tienen efectos sobre el empleo que difieren en cada caso de ciudades. Así, las actividades ligadas a la minería -y en específico al cobre- tienen un rol clave en la estructura de la economía urbana de las ciudades en auge, lo cual contrasta con el panorama de las ciudades en una 
situación sin auge, donde la minería pierde este rol protagónico, destacando en este sentido las actividades ligadas a la agricultura y, de forma relevante, aquellas relacionadas con la industria no metálica. Es de especial importancia destacar el papel de la industria no metálica, dado que en general se considera a Chile un país poco industrializado; sin embargo, la industria no metálica representa, en primer lugar, actividades de procesamiento de commodities (celulosa, productos agrícolas procesados). Si bien a escala nacional tiene una relevancia claramente menor en comparación con la minería para el empleo en servicios, en las ciudades sin auge esta industria tiene un papel clave. Por su parte, los efectos de la inversión nacional sobre el empleo muestran tendencias que guardan relación con un patrón de comportamiento diferente en cada grupo de ciudades, ya que en aquellas ciudades en una situación de auge, la inversión tiende a focalizarse en el sector de hotelería/restaurantes, mientras que en las ciudades sin auge, los efectos de la inversión nacional actúan de forma importante sobre el empleo en construcción y comercio.

\section{Reflexiones finales}

En primer lugar, es posible constatar, por una parte, que las ciudades en auge presentan pautas de crecimiento muy diferentes de las que muestran las demás ciudades; y por otra, que el fundamento de este crecimiento es estructuralmente diferente en uno y otro caso. El crecimiento de las actividades no exportadoras se relaciona con la exportación, evidenciando, además, que estos rubros están relacionados entre sí: particularmente entre construcción y hotelería hay retroalimentaciones positivas. Se puede concluir, entonces, un efecto positivo de la actividad exportadora sobre los servicios no exportables en ciudades en auge. Considerando que otros estudios han identificado "enclaves mineros", demostrando la débil vinculación directa de la actividad minera con la economía local (Arias, Atienza \& Cademartori, 2014), es importante destacar que el impulso de la minería sobre la economía local se genera en buena parte por el empleo y las remuneraciones obtenidos en el sector en auge. Debido a esto, parece ser que el número de empleados y sus ingresos son el principal impulso sobre los rubros no exportadores, lo que en términos teóricos implica que el papel de los empleados en la evaluación de los efectos del síndrome holandés, como también en la evaluación de los "enclaves", tiene que ser conceptualmente enfatizado. Los efectos secundarios y de retroalimentación asociados a los hogares partícipes del auge aparentemente son de importancia mayor en comparación con el efecto directo de la exportación.

Para las ciudades sin auge, se observa un destacado papel de la industria no metálica, un sector menos protagónico en la exportación chilena para el crecimiento del empleo en servicios. En términos teóricos, esto significa que se debería examinar cómo estos efectos traspasan las escalas. Si el auge exportador del cobre significa un freno para la exportación de commodities agrícolas, tal como lo plantea la tesis del síndrome holandés, implicaría que también puede frenar el sector de servicios en las economías urbanas en regiones agrícolas. Sumado a esto, se demuestra el papel de la inversión pública, sobre todo del FNDR y de la inversión municipal, lo cual -en términos teóricos- significa la necesidad de incluir el gasto público en la 
argumentación sobre cómo los efectos del auge exportador traspasan las escalas, particularmente en la discusión de un efecto de redistribución espacial de renta exportadora sobre las economías urbanas sin auge.

Finalmente, en cuanto a la política pública y la importancia de diferentes actores económicos, vale destacar, por una parte, la importancia de los mecanismos que hacen partícipes a distintos hogares y distintas regiones de un auge exportador específico, ya que los resultados muestran el papel destacado de los empleados y de las remuneraciones que ellos reciben para las economías urbanas de las ciudades en auge; y por otra, la importancia del gasto público en las ciudades sin auge.

\section{Referencias bibliográficas}

Agacino, R., González, C. \& Rojas, J. (1998). Capital transnacional y trabajo. El desarrollo minero en Chile. Santiago de Chile: LOM ediciones.

Alvear, J. (1975). Chile: nuestro cobre. Santiago de Chile: Editorial Lastra.

Arias, M., Atienza, M. \& Cademartori, J. (2014). Large mining enterprises and regional development in Chile: Between the enclave and cluster. Journal of Economic Geography, 14(1), 73-95. https://doi.org/10.1093/jeg/lbt007

Aroca, P. \& Atienza, M. (2008). La conmutación regional en Chile y su impacto en la Región de Antofagasta. EURE Revista Latinoamericana de Estudios Urbano Regionales, 34(102), 97-121. http://dx.doi.org/10.4067/S0250-7161200800020000

Atienza, M., Lufin, M., Soto, J. \& Cortés, Y. (2015). ¿Es la región de Antofagasta un caso exitoso de desarrollo local basado en la minería? En C. Rodríguez, M. Atienza, M. Lufin, G. Romaní, J. A. González, R. González, ... J. Soto, Sistemas, coaliciones, actores $y$ desarrollo económico territorial en regiones mineras. Innovación territorial aplicada (pp. 97-117). Antofagasta: Universidad Católica del Norte.

Banco Central de Chile (2016). Estadisticas monetarias y financieras, 2016. En http://si3. bcentral.cl/estadisticas/Principal1/Informes/EMF/AGREGADOS_MONETARIOS/ Informes.html

Barrera, M. (1973). El conflicto obrero en el enclave cuprifero. Santiago de Chile: Instituto de Economía y Planificación, Universidad de Chile.

Barrett, S. (1994). Paradise: Class, commuters, and ethnicity in rural Ontario. Toronto: University of Toronto.

Bell, M. \& Brown, D. (2006). Who are the visitors? Characteristics of temporary movers in Australia. Population, Space and Place, 12(2), 77-92. http://doi.org/10.1002/psp.390

Cámara Chilena de la Construcción (cchc). (2015). Comunicaciones. En http://www.cchc. $\mathrm{cl} /$ comunicaciones/noticias/84785-en-un-600-aumento-la-inversion-inmobiliariadurante-la-ultima-decada-

Comisión Chilena del Cobre (cochilco). (2016). Estadísticas Producción Minera, 2016. En http://www.cochilco.cl/estadisticas/produccion.asp

Corden, W. M. \& Neary, J. P. (1982). Booming sector and de-industrialization in a small open economy. The Economic Journal, 92(368), 825-848. http://dx.doi. org $/ 10.2307 / 2232670$ 
Corden, W. M. (1984). Booming sector and Dutch disease economics: Survey and consolidation. Oxford Economic Papers. New Series, 36(3), 359-380. https://doi. org/10.1093/oxfordjournals.oep.a041643

Daher, A. (2003). Regiones-commodities: Crisis y contagio en Chile. EURE Revista Latinoamericana de Estudios Urbano Regionales, 29(86), 89-108. http://dx.doi. org/10.4067/S0250-71612003008600005

De Mattos, C. (2002). Mercado metropolitano de trabajo y desigualdades sociales en el Gran Santiago: ¿Una ciudad dual? EURE Revista Latinoamericana de Estudios Urbano Regionales, 28(85), 51-70. http://dx.doi.org/10.4067/S0250-71612002008500004

Durán, H. (1990). Impacto ambiental de la actividad minera en Chile: Balance preliminar. Ambiente y Desarrollo, 6(1), 65-76. En http://www.cipma.cl/web/200.75.6.169/ RAD/1990/1_Duran.pdf

Echeverría, M. \& López, D. (2004). Flexibilidad laboral en Chile: Las empresas y las personas. Cuadernos de Investigación 22. Santiago de Chile: Departamento de Estudios, Dirección del Trabajo. En http://www.inspecciondeltrabajo.cl/1601/articles-74726_ recurso_1.pdf

El Mercurio de Antofagasta (2011). Los inmigrantes cambian el rostro del norte chileno. Recuperado el 12 de enero de 2016 desde http://www.mercurioantofagasta.cl/ prontus4_noticias/site/artic/20110605/pags/20110605023503.html

Encuesta CaSEn 2013. Véase Ministerio de Desarrollo Social, 2013.

Ennis, G., Finlayson, M. \& Speering, G. (2013). Expecting a boomtown? Exploring potential housing-related impacts of large scale resource developments in Darwin. Human Geographies, 7(1), 33-42. En http://humangeographies.org.ro/articles/71/7_1_13_3_ ennis.pdf

Fox Gotham, K. (2009). Creating liquidity out of spatial fixity: The secondary circuit of capital and the subprime mortgage crisis. International Journal of Urban and Regional Research, 33(2), 355-371. http://doi.org/10.1111/j.1468-2427.2009.00874.x

Fuentes, L. \& Link, F. (2014). Competitividad, mercados del trabajo y estructura socio territorial en Bogotá, Lima y Santiago. Revista de Geografía Norte Grande, 59, $105-$ 122. http://dx.doi.org/10.4067/S0718-34022014000300007

Garcés, E. (2003). Las ciudades del cobre: Del campamento de montaña al hotel minero como variaciones de la company-town. EURE Revista Latinoamericana de Estudios Urbano Regionales, 29(88), 131-148. http://dx.doi.org/10.4067/S0250-71612003008800006

García-Huidobro, A. \& Maragaño, A. (2010). La vertebración territorial en regiones de alta especialización: Valle Central de Chile. Alcances para el desarrollo de zonas rezagadas en torno a los recursos naturales. EURE Revista Latinoamericana de Estudios Urbano Regionales, 36(107), 49-65. http://dx.doi.org/10.4067/S0250-71612010000100003

Harvey, D. (2008). El derecho a la ciudad. New Left Review, 53, 23-39. En http://newleftreview. es/authors/david-harvey

Harvey, D. (2011). Roepke Lecture in Economic Geography - Crises, geographic disruptions and the uneven development of political responses. Economic Geography, 87(1), 1-22. http://doi.org/10.1111/j.1944-8287.2010.01105.x

Haslam McKenzie, F. M. \& Rowley, S. (2013). Housing market failure in a booming economy. Housing Studies, 28(3), 373-388. http://doi.org/10.1080/02673037.2013.759177 
Houghton, D. S. (1993). Long distance commuting: A new approach to mining in Australia. The Geographical Journal, 159(3), 281-290. http://doi.org/10.2307/3451278

Howitt, R. (2001). Rethinking resource management: Justice, sustainability and indigenous peoples. Londres: Routledge.

Instituto Nacional de Estadística (INE), Chile(2015). Comunas: Población estimada al 30 de junio por sexo y edad simple 2002 - 2020. Base de datos. En http://www.ine.cl/ estadisticas/demograficas-y-vitales

Jamett, I. \& Paredes, D. (2013). Conmutación de larga distancia en Chile: Estimando el premio por trabajar muy lejos de casa. Estudios de Economía, 40(2), 179-209. http:// dx.doi.org/10.4067/S0718-52862013000200004

Jeftanovic, P. (1992). El síndrome holandés. Teoría, evidencia y aplicación al caso chileno (1901-1940). Estudios Públicos, (45), 299-331. En https://www.cepchile.cl/cep/site/ artic/20160303/asocfile/20160303184228/rev45_jeftanovic.pdf

Lagos, G., Blanco, H., Torres, V. \& Bustos, B. (2001). Minería, minerales y desarrollo sustentable en Chile. En Equipo mmsD América del Sur, Minería, minerales y desarrollo sustentable en América del Sur (pp. 341-440). Santiago de Chile / Montevideo: Centro de Investigación y Planificación del Medio Ambiente (CIPMA) y Centro Internacional de Investigaciones para el Desarrollo (IDRC) - Iniciativa de Investigación sobre Políticas Mineras (IIPM). En http://bit.ly/2tHqpqk

Lawrie, M., Tonts, M. \& Plummer, P. (2011). Boomtowns, resource dependence and socioeconomic well-being. Australian Geographer, 42(2), 139-164. http://dx.doi.org/10.10 80/00049182.2011.569985

Lozeva, S. \& Marinova, D. (2010). Negotiating gender: Experience from Western Australian mining industry. Journal of Economic and Social Policy, 13(2), 177-209. En http:// epubs.scu.edu.au/cgi/viewcontent.cgi?article=1143\&context=jesp

Mcintosh, A. (2012). Thinking space: ten truths about Australia's rush to mine and the mining workforce. Australian Geographer, 43(4) 331-337. http://dx.doi.org/10.1080/000491 82.2012.731297

Ministerio de Desarrollo Social, Gobierno de Chile (2013). Resultados Encuesta Casen 2013. Presentaciones sectoriales: Trabajo, Educación, Salud y Vivienda / Grupos de Población: Inmigrantes; Pueblos Indígenas; Adultos Mayores; Género; Niños, Niñas y Adolescentes; Personas con Dificultades y/o Condiciones Permanentes y de Larga Duración. En http://www.ministeriodesarrollosocial.gob.cl/resultados-encuesta-casen-2013/

Ministerio de Desarrollo Social, Gobierno de Chile (2014). Serie de Inversión Pública Regionalizada 2001-2015. En http://sni.ministeriodesarrollosocial.gob.cl/centro-dedocumentacion/publicaciones/

Ministerio de Transportes y Telecomunicaciones, Gobierno de Chile (2015). Tráfico entre pares de ciudades en el año 2015. En www.jac.gob.cl/wp-content/../2015-Trafico-entrepares-de-ciudades-Nacional.xls

Organization for Economic Co-operation and Development (OECD). (2012). Working party on territorial policy in urban areas. National urban policy reviews. The case of Chile. París: OECD Publishing.

Park, M. \& Stokowski, P. (2009). Social disruption theory and crime in rural communities: comparisons across three levels of tourism growth. Tourism Management, 30(6), 905915. https://doi.org/10.1016/j.tourman.2008.11.015 
Rehner, J. \& Vergara, F. (2014). Efectos recientes de la actividad exportadora sobre la reestructuración urbana en Chile. Revista de Geografía Norte Grande, (59), 83-103. http://dx.doi.org/10.4067/S0718-34022014000300006

Rehner, J., Baeza, S. \& Barton, J. (2014). Chile's resource-based export boom and its outcomes: regional specialization, export stability and economic growth. Geoforum, 56, 35-45. https://doi.org/10.1016/j.geoforum.2014.06.007

Romero, H. \& Vásquez, A. (2005). La comodificación de los territorios urbanizables y la degradación ambiental en Santiago de Chile. Scripta Nova. Revista Electrónica de Geografía y Ciencias Sociales, 9(194-68). En http://www.ub.edu/geocrit/sn/sn-194-68.htm

Sánchez, J. \& Huepe, C. (1995). Minería y medioambiente en Chile: Situación ambiental y legal. Documento preparado como parte del Proyecto "Growth, the Environment and Fiscal Policy in the Chilean Mining Sector", financiado por IDRc. Santiago, Chile.

Sennett, R. (2009). La corrosión del carácter: las consecuencias personales del trabajo en el nuevo capitalismo. Barcelona: Anagrama.

Servicio de Impuestos Internos (siI), Chile (2015). Estadísticas de empresas por rubro, región y comuna (2005-2014). En http://www.sii.cl/estadisticas/empresas_rubro.htm

Spies, M. (2006). Distance between home and workplace as a factor for job satisfaction in the North-West Russian oil industry. Fennia - International Journal of Geography, 184(2), 133-149. En https://fennia.journal.fi/article/view/3724

Storey, K. (2001). Fly-in, fly-out and fly-over: mining and regional development in Western Australia. Australian Geographer, 32(2), 133-148. http://dx.doi. org/10.1080/00049180120066616

Storey, K. (2009). The evolution of commute work in Canada and Australia. Biography, shiftlabour and socialisation in a Northern industrial city - The Far North: Particularities of labour and human socialisation. Conference proceedings, Novy Urengoy, Russia. December 4-6 2008, 23-32.

Storey, K. \& Shrimpton, M. (1988). Long-distance commuting in the Canadian mining industry. Working Paper, 43, Centre for Resource Studies Queen's University, Kingston, on, Canada.

Thodes Miranda, E. (2016). Segregación socioespacial en ciudades mineras: el caso de Antofagasta, Chile. Notas de población, 43(102), 203-227 [CEPAL, LC/G.2673-P]. En http://repositorio.cepal.org/handle/11362/40265

Van Wijnbergen, S. (1984). The "Dutch Disease": A disease after all? The Economic Journal, 94(373), 41-55. http://doi.org/10.2307/2232214

Wilkinson, K., Reynolds, R., Thompson, J. \& Ostresh, L. (1982). Local social disruption and Western energy development. Pacific Sociological Review, 25(3), 275-296. http://doi. org/10.2307/1388767

Williams, C. (1981). Open cut: The working class in an Australian mining town. Series Studies in Society, 8. Sydney: George Allen \& Unwin.

Zapata, F. (1977). Enclaves y sistemas de relaciones industriales en América Latina. Revista Mexicana de Sociología, 39(2), 719-731. http://doi.org/10.2307/3539782

Zapata, F. (2001). El trabajo en la vieja y en la nueva economía. En E. de la Garza \& J. Neffa (eds.), El futuro del trabajo. El trabajo del futuro (pp. 99-110). Buenos Aires: Consejo Latinoamericano de Ciencias Sociales (CLACSO). En http://bibliotecavirtual.clacso.org. ar/clacso/gt/20101102090632/4zap.pdf 
